\title{
Hausdorff dimensions in Engel expansions
}

\author{
by \\ YAN-YAN LiU and Jun Wu (Wuhan)
}

1. Introduction. Given $x$ in $(0,1]$, let $x=\left[d_{1}(x), d_{2}(x), \ldots\right]$ denote the Engel expansion of $x$, that is,

$$
x=\frac{1}{d_{1}(x)}+\frac{1}{d_{1}(x) d_{2}(x)}+\ldots+\frac{1}{d_{1}(x) d_{2}(x) \ldots d_{n}(x)}+\ldots,
$$

where $\left\{d_{j}(x), j \geq 1\right\}$ is a sequence of positive integers satisfying $d_{1}(x) \geq 2$ and $d_{j+1}(x) \geq d_{j}(x)$ for $j \geq 1$ (see [3]). In [3], János Galambos proved that for almost all $x \in(0,1]$,

$$
\lim _{n \rightarrow \infty} d_{n}^{1 / n}(x)=e .
$$

Also he posed the following questions (see [3], P132):

(i) Find the Hausdorff dimension of the set where (2) fails.

(ii) For any $k \geq 1$, let

$$
A_{k}=\left\{x \in(0,1]: \log d_{n}(x) \geq k n \text { for any } n \geq 1\right\} .
$$

Find the Hausdorff dimension of the set $A_{k}$.

For (i), the second author [4] has proved that the Hausdorff dimension of the set where (2) fails is 1 .

In this paper, we get a stronger result than those in (i) and (ii). We show

Theorem. For any $\alpha \geq 1$, let

$$
A(\alpha)=\left\{x \in(0,1]: \lim _{n \rightarrow \infty} d_{n}^{1 / n}(x)=\alpha\right\} .
$$

Then

$$
\operatorname{dim}_{\mathrm{H}} A(\alpha)=1 .
$$

As corollaries of the Theorem, both the Hausdorff dimensions in (i) and (ii) are 1.

2000 Mathematics Subject Classification: Primary 11K55; Secondary 28A80.

Project supported by the Special Funds for Major State Basic Research Projects. 
We use $|\cdot|$ to denote the diameter of a subset of $(0,1], \operatorname{dim}_{\mathrm{H}}$ to denote the Hausdorff dimension, [ ] the integer part of a real number and cl the closure of a subset of $(0,1]$ respectively.

2. Proof of the Theorem. The aim of this section is to prove the main result of this paper.

In what follows we often make use of the code space. Let $\left\{M_{n}, n \geq 1\right\}$ be a sequence of positive numbers such that $M_{1}>1, M_{k}<M_{k+1}$ for any $k \geq 1$. For any $n \geq 1$, let

$$
D_{n}=\left\{\left(\sigma_{1}, \ldots, \sigma_{n}\right) \in \mathbb{N}^{n}: k M_{k}<\sigma_{k} \leq(k+1) M_{k} \text { for all } 1 \leq k \leq n\right\} .
$$

Define

$$
D=\bigcup_{n=0}^{\infty} D_{n} \quad\left(D_{0}=\emptyset\right) .
$$

For any $\sigma=\left(\sigma_{1}, \ldots, \sigma_{n}\right) \in D_{n}$, we use $J_{\sigma}$ to denote the following closed subinterval of $(0,1]$ :

$$
J_{\sigma}=\bigcup_{k=\left[(n+1) M_{n+1}\right]+1}^{\left[(n+2) M_{n+1}\right]} \operatorname{cl}\left\{x \in(0,1]: d_{1}(x)=\sigma_{1}, \ldots, d_{n}(x)=\sigma_{n}, d_{n+1}(x)=k\right\},
$$

and call it an $n$-order interval.

Define

$$
E=\bigcap_{n=0}^{\infty} \bigcup_{\sigma \in D_{n}} J_{\sigma}
$$

It is obvious that

$$
E=\left\{x \in(0,1]: n M_{n}<d_{n}(x) \leq(n+1) M_{n} \text { for all } n \geq 1\right\} .
$$

Proof of the Theorem. We divide the proof into two parts:

PART I: $\alpha>1$. For any $n \geq 1$, let $M_{n}=\alpha^{n}$. Now we estimate the length of $J_{\sigma}$ for any $\sigma \in D_{n}$. Since for any $(n+1) \alpha^{n+1}<k \leq(n+2) \alpha^{n+1}$,

$$
\begin{aligned}
\mid\left\{x \in(0,1]: d_{1}(x)=\sigma_{1}, \ldots, d_{n}(x)=\sigma_{n},\right. & \left.d_{n+1}(x)=k\right\} \mid \\
& =\frac{1}{\sigma_{1} \ldots \sigma_{n}}\left(\frac{1}{k-1}-\frac{1}{k}\right),
\end{aligned}
$$

we have

$$
\left|J_{\sigma}\right|=\sum_{k=\left[(n+1) M_{n+1}\right]+1}^{\left[(n+2) M_{n+1}\right]} \frac{1}{\sigma_{1} \ldots \sigma_{n}}\left(\frac{1}{k-1}-\frac{1}{k}\right) .
$$

Therefore

$$
(n+2)^{-(n+2)} \alpha^{-(n+1)(n+2) / 2} \alpha^{-(n+1)} \leq\left|J_{\sigma}\right| \leq \alpha^{-(n+1)(n+2) / 2} .
$$


Let $\mu$ be a mass distribution supported on $E$ such that for any $n \geq 0$ and $\sigma \in D_{n}$,

$$
\mu\left(J_{\sigma}\right)=\frac{1}{\sharp D_{n}} \quad\left(\sharp D_{0}=1\right) .
$$

By the definition of $D_{n}$, it is easy to check that

$$
c^{-n} \alpha^{n(n+1) / 2} \leq \sharp D_{n} \leq c^{n} \alpha^{n(n+1) / 2},
$$

where $c$ is a positive constant which does not depend on $n$.

For any $x \in E$, we prove that

$$
\liminf _{r \rightarrow 0} \frac{\log \mu(B(x, r))}{\log r} \geq 1
$$

where $B(x, r)$ denotes the open ball with center at $x$ and radius $r$.

For $r<\alpha^{-3}$, choose $n \geq 3$ such that

$$
\alpha^{-n(n+1) / 2}<r \leq \alpha^{-(n-1) n / 2} .
$$

By (5), $B(x, r)$ can intersect at most $4 n^{n} \alpha^{n-1}(n-2)$-order intervals, thus by (6) and (7),

$$
\liminf _{r \rightarrow 0} \frac{\log \mu(B(x, r))}{\log r} \geq \liminf _{n \rightarrow \infty} \frac{\log \left(c^{n-2} \alpha^{-(n-2)(n-1) / 2} 4 n^{n} \alpha^{n-1}\right)}{\log \alpha^{-n(n+1) / 2}}=1 .
$$

By [2], Proposition 2.3, (see also [1], Proposition 4.9) we have $\operatorname{dim}_{\mathrm{H}} E=1$. Since $E \subset A(\alpha)$, we have $\operatorname{dim}_{\mathrm{H}} A(\alpha)=1$.

PART II: $\alpha=1$. The proof of this part is very similar to Part I; we just give an outline.

For any $n \geq 1$, let

$$
M_{n}=\left(1+\frac{1}{\sqrt{n}}\right)^{n} .
$$

Then as in Part I, we have

$$
\begin{gathered}
(n+2)^{-(n+2)}\left(\prod_{k=1}^{n+1}\left(1+\frac{1}{\sqrt{k}}\right)^{k}\right)^{-1}\left(1+\frac{1}{\sqrt{n+1}}\right)^{-(n+1)} \\
\leq\left|J_{\sigma}\right| \leq\left(\prod_{k=1}^{n+1}\left(1+\frac{1}{\sqrt{k}}\right)^{k}\right)^{-1}, \\
c^{-n} \prod_{k=1}^{n}\left(1+\frac{1}{\sqrt{k}}\right)^{k} \leq \sharp D_{n} \leq c^{n} \prod_{k=1}^{n}\left(1+\frac{1}{\sqrt{k}}\right)^{k} .
\end{gathered}
$$

For any $x \in E, r<\left(\prod_{k=1}^{3}(1+1 / \sqrt{k})^{k}\right)^{-1}$, choose $n \geq 3$ such that

$$
\left(\prod_{k=1}^{n}\left(1+\frac{1}{\sqrt{k}}\right)^{k}\right)^{-1}<r \leq\left(\prod_{k=1}^{n-1}\left(1+\frac{1}{\sqrt{k}}\right)^{k}\right)^{-1} \text {. }
$$


By $(10), B(x, r)$ can intersect at most $4 n^{n}(1+1 / \sqrt{n-1})^{n-1}(n-2)$-order intervals, thus by (6) and (11), we have

$$
\begin{aligned}
& \liminf _{r \rightarrow 0} \frac{\log \mu(B(x, r))}{\log r} \\
& \geq \liminf _{n \rightarrow \infty} \frac{\log \left(c^{n-2}\left(\prod_{k=1}^{n-2}\left(1+\frac{1}{\sqrt{k}}\right)^{k}\right)^{-1} 4 n^{n}\left(1+\frac{1}{\sqrt{n-1}}\right)^{n-1}\right)}{\log \left(\prod_{k=1}^{n}\left(1+\frac{1}{\sqrt{k}}\right)^{k}\right)^{-1}} .
\end{aligned}
$$

Since $\left\{(1+1 / \sqrt{n})^{\sqrt{n}}, n \geq 1\right\}$ is an increasing sequence such that for any $n \geq 1$,

$$
2 \leq\left(1+\frac{1}{\sqrt{n}}\right)^{\sqrt{n}} \leq e
$$

and

$$
1+\frac{1}{\sqrt{2}}+\frac{1}{\sqrt{3}}+\ldots+\frac{1}{\sqrt{n}} \geq \int_{1}^{n} x^{-1 / 2} d x=2 n^{1 / 2}-2
$$

we have

$$
\liminf _{r \rightarrow 0} \frac{\log \mu(B(x, r))}{\log r} \geq 1
$$

completing the proof of the Theorem.

Corollary 1. For any $k \geq 1, \operatorname{dim}_{\mathrm{H}} A_{k}=1$.

Proof. For any $k \geq 1$, choose $M>e^{k}$. Let $M_{n}=M^{n}$ for any $n \geq 1$. Then $E \subset A_{k}$. By the proof of the Theorem, we have $\operatorname{dim}_{\mathrm{H}} E=1$, thus $\operatorname{dim}_{\mathrm{H}} A_{k}=1$.

From the proof of the Theorem, we can also get the following corollaries immediately.

Corollary 2. For any $n \geq 2$ and $\alpha \geq 1$, let

$$
B(\alpha)=\left\{x \in(0,1]: \lim _{n \rightarrow \infty} \frac{d_{n+1}(x)}{d_{n}(x)-1}=\alpha\right\} .
$$

Then

$$
\operatorname{dim}_{\mathrm{H}} B(\alpha)=1 \text {. }
$$

Corollary 3. The Hausdorff dimension of the set where (2) fails is 1.

Acknowledgments. The authors thank the referee for his (her) valuable comments and Dr. De-jun Feng for his helpful suggestions. 


\section{References}

[1] K. J. Falconer, Fractal Geometry. Mathematical Foundations and Applications, Wiley, 1990.

[2] - Techniques in Fractal Geometry, Wiley, 1997.

[3] J. Galambos, Reprentations of Real Numbers by Infinite Series, Lecture Notes in Math. 502, Springer, 1976.

[4] J. Wu, A problem of Galambos on Engel expansions, Acta Arith. 92 (2000), 383-386.

Department of Mathematics and Center of Non-linear Science

Wuhan University

430072, Wuhan

People's Republic of China

E-mail: yyliu@nlsc.whu.edu.cn

wujunyu@public.wh.hb.cn

Received on 26.6.2000

and in revised form on 21.11.2000 